\title{
Serum ischemia modified albumin (IMA) levels of patients with bipolar disorder in remission and healthy controls.
}

\author{
Kubranur Unal $^{1 *}$, Canan Topcuoglu ${ }^{2}$, Merve Cingi Yirun ${ }^{3}$ \\ ${ }^{1}$ Department of Biochemisty, Ankara Polatli Public Hospital, Ankara, Turkey \\ ${ }^{2}$ Department of Biochemisty, Ankara Numune Training and Research Hopital, Ankara, Turkey \\ ${ }^{3}$ Department of Psychiatry, Bartın Public Hospital, Bartın, Turkey
}

\begin{abstract}
Oxidative stress might play an important role in neurological and psychiatric diseases due to the central nervous system sensitivity to reactive oxygen species. Aim of this study is to contribute these data about oxidative stress in bipolar disorder, by detecting IMA levels of bipolar disorder patients in remission and also by comparing these results with healthy controls. Study population consisted of 35 outpatients with bipolar disorder, who being in remission. The patients meeting the Diagnostic and Statistical Manual of Mental Disorders, $5^{\text {th }}$ Edition criteria for bipolar disorder I. 36 healthy subjects were included as control group. Serum ischemia modified albumin levels of all participants were determined and the results were compared between these two groups. The age, gender and ischemia modified albumin levels were not significantly different between bipolar disorder patients and healthy controls. Studies focusing on oxidative stress in bipolar disorder have reached controversial results up till now. The many different markers have been investigated to explain the effects of oxidative stress on bipolar disorders, and this might be reason why there are conflicting results. In addition, the past episodes of patients who were in remission during this study are unclear. Further investigations are needed to be carrying out to illustrate possible changes of oxidative balance in psychiatric disorders, including bipolar disorder.
\end{abstract}

Keywords: Bipolar disorder, Ischemia modified albumin (IMA), Oxidative stress.

Accepted on May 29, 2018

\section{Introduction}

Bipolar disorder is one of the complex psychiatric disorders courses with episodes of mania/hypomania, depression and mixed [1]. Since its pathophysiology cannot be determined certainly, oxidative stress and inflammatory processes are one of several important factors under investigation along with many other possible etiological mechanisms [2].

Oxidative status is described as a balance between the development and inactivation of reactive oxygen species (ROS) [3]. Any increase in the rate of ROS development, or decrease in their inactivation, may disrupt this balance, resulting in oxidative damage. Ischemia modified albumin (IMA) is a biomarker, which is formed as a consequence of modification of albumin by ROS. Consequently the elevations in ROS may modify the N-terminal portion of albumin causing an increased formation of IMA. These different levels of IMA in the serum may be used as a marker to oxidative stress [4].

Since recent data showed variable results about the complex role of biological and environmental factors in the etiology of affective disorders; last studies started to investigate oxidative stress, which can be altering nerve cell composition and take part in pathophysiology [5]. To the best of our knowledge, this is the first study reporting the analysis of IMA levels on bipolar disorder patients in remission. Aim of our study is to contribute these data about oxidative stress in bipolar disorder, by detecting IMA levels of bipolar disorder patients in remission and also by comparing these results with healthy controls.

\section{Methods}

\section{Participants}

Our study's sample consisted of 35 outpatients with bipolar disorder, who being in remission (RE). The outpatients who had applied to the psychiatry department outpatient clinic between June 2015 and December 2015 and who met Diagnostic and Statistical Manual of Mental Disorders, $5^{\text {th }}$ Edition (DSM-5) criteria for bipolar disorder I. 36 healthy individuals, who were working as hospital staff and had no prior psychiatric history or on-going complaints, formed the control group (HC). For the entire sample, inclusion criteria were an age between 18 and $65 \mathrm{y}$ and no history of dementia or cognitive deterioration. The participants with any acute or 
chronic disease or medication or any addiction except smoking during the study were not included in the study.

Two psychiatrists discussed the patients to confirm that the outpatients $(n=35)$ did not meet the DSM-5 criteria of any bipolar disorder episode (depressive, manic, or hypomanic) for at least 6 months and that the control group participants $(n=36)$ had no proof of a present or previous psychiatric disease. The Hamilton Depression Rating Scale (HAM-D) and Young Mania Rating Scale (YMRS) were also used to support the diagnoses of RE (i.e., HAM-D score $<7$ or YMRS score $<4$ ).

This study was approved by the Ethical Committee from the research office of Ankara Polatli Public Hospital. All the participants gave informed consent for participation in the study.

\section{Instruments}

Hamilton depression rating scale (HAM-D): The original version of the scale, which was conceived by Hamilton in 1960, contains 17 items, each of which is scored as $0-4$; the maximum total score is 53 . The patients were scored with the 17-item version in this study. Williams advanced a recent version of the HDRS to improve the inter-rater reliability (Structured Interview for Hamilton Depression Rating Scale-21) [6]. The Turkish version of the scale was reported to be valid and reliable [7]. In this study patients, were evaluated with the 17 -item version.

Young mania rating scale (YMRS): YMRS is an 11-item diagnostic criterion used to evaluate the severity of mania. Each scale item measures 5 degrees of severity. The Turkish version of the scale was reported to be valid and reliable [8].

\section{Biochemical analysis}

Venous blood samples were drawn in the morning around 8 am into gel containing tubes and centrifuged at $1300 \mathrm{~g}$ for $10 \mathrm{~min}$ to analyze the separated sera. The serums were stored at $-80^{\circ} \mathrm{C}$ until the day of analysis. Haemolysed and icteric serums were not used in this study.

IMA was evaluated by a colorimetric assay developed by BarOr et al. based on measurement of unbound cobalt after incubation with serum [9]. Elevated amounts of IMA results in less cobalt binding and more residual unbound cobalt available for complex with a chromogen (dithiothreitol (DTT)), which can be measured photometrically; the procedure was as follows: $50 \mu \mathrm{L}$ of $0.1 \%$ cobalt chloride (Merck KGaA, Darmstadt, Germany) was added to $200 \mu \mathrm{L}$ of serum, gently mixed, and waited $10 \mathrm{~min}$ for adequate cobalt-albumin binding. 50 microliters of DTT (Merck KGaA, Darmstadt, Germany), at a concentration of $1.5 \mathrm{mg} / \mathrm{ml}$, was added as a colorizing agent and the reaction was stopped 2 min later by adding $1.0 \mathrm{~mL}$ of $0.9 \% \mathrm{NaCl}$. The colored product was measured using a spectrophotometer at $470 \mathrm{~nm}$ (Shimadzu, UV1601, and Japan) and compared to a serum cobalt blank without DTT and reported in absorbance units (ABSU).

\section{Statistical analysis}

Statistical Package for the Social Sciences (SPSS) 20.0 was used for the analysis. The Kolmogorov-Smirnov test was used to determine whether the parameters were normally distributed. Results were expressed as mean and standard deviations (SD). The two groups (RE and HC) were examined with the paired ttest. A p-value of less than 0.05 was considered to be statistically significant.

\section{Results}

There were 35 patients (16 males, 19 females) in the RE group, 36 (14 males, 22 females) in the HC group, with mean ages of $38.2 \pm 7.8$ and $33.7 \pm 6.3 \mathrm{y}$, respectively. The results were compared between these two groups. The age, gender and IMA levels of the 2 groups are shown in Table 1.

Table 1. Comparison of laboratory findings between two groups.

\begin{tabular}{llll}
\hline & $\mathbf{R E}(\mathbf{n}=\mathbf{3 5})$ & $\mathbf{H C}(\mathbf{n}=\mathbf{3 6})$ & P value \\
\hline Age & $38.2 \pm 7.8$ & $33.7 \pm 6.3$ & 0.86 \\
\hline Male/female ratio & $16 / 19$ & $14 / 22$ & 0.12 \\
\hline IMA (ABSU) & $0.532 \pm 0.14$ & $0.546 \pm 0.128$ & 0.48 \\
\hline
\end{tabular}

ABSU: Absorbance Unit; IMA: Ischemia Modified Albumin.

There was no significant difference between both group, in terms of age, gender and IMA levels $(\mathrm{p}>0.05)$. Figure 1 shows IMA levels in $\mathrm{RE}$ and $\mathrm{HC}$ groups.

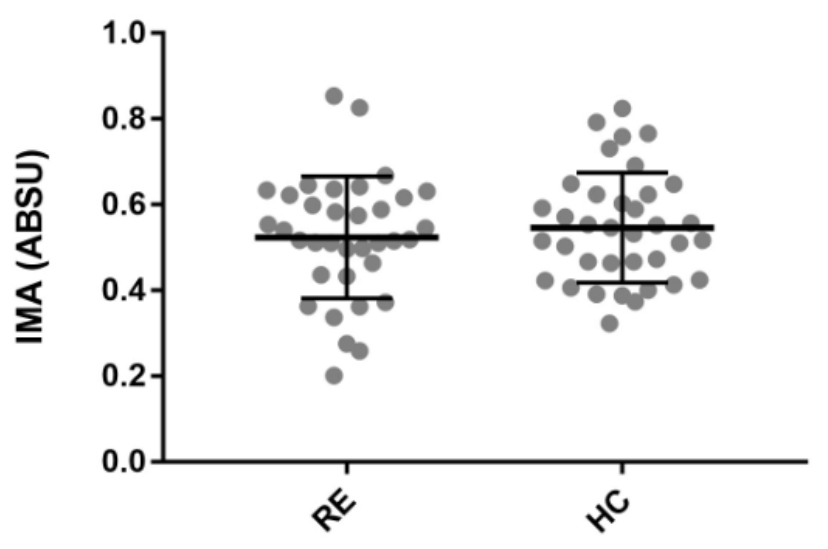

Figure 1. IMA levels in RE and Hc groups.

\section{Discussion}

Oxidative stress plays an important role in the pathophysiology of many diseases, particularly in neurological and psychiatric diseases due to the central nervous system sensitivity to ROS [10]. There is evidence that IMA increases in diseases associated with ischemia and oxidative stress [11,12]. The induced ROS modify the N-terminal portion of albumin causing an increased formation of IMA. In other words, IMA is formed as a consequence of modification of albumin by ROS [4]. 
In several studies, the role of oxidative imbalance in the etiological of bipolar disorder has investigated [13-19]. However, evidence about oxidative stress in bipolar disorder is controversial. Some studies shown that oxidative stress might be involved in the pathophysiology of bipolar disorder; in a study, Na+-K+-ATPase activity and serum Thiobarbituric Acid Reactive Substances (TBARS) levels were significantly decreased and increased respectively in RE when compared to the $\mathrm{HC}$ [13]. In an another study conducted on patients with bipolar disorder in remission, Nitric Oxide (NO) and Superoxide Dismutase (SOD) levels found to be higher than in the HC [14]. There is also a study; Lipid Hydroperoxides (LPH) levels were found significantly high than in HC [15].

The current study reports that IMA levels were not significantly different between patients with bipolar disorder in $\mathrm{RE}$ and HC. These results show consistency with the findings of some prior studies researching oxidative stress markers in bipolar disorder. In a study, blood levels of Total Antioxidant Status (TAS), Total Oxidant Status (TOS), and Oxidative Stress Index (OSI), which are other molecules indicating oxidative stress, were showed not to differ significantly between the bipolar disorder patients in RE and HC [16]. In another study, SOD levels could be detected no significant difference between RE and HC, but Thiobarbituric Acid Reactive Substances (TBARS) levels were significantly higher in RE compared to HC [17]. Results of another study demonstrated that SOD and S100B levels were found no significant changes in RE when compared to HC [18]. There is also a study, total radicaltrapping antioxidant parameter (TRAP) and TBARS were found no significant changes between RE and HC [19].

Studies focusing on oxidative stress in bipolar disorder have reached controversial results up till now. The many different markers have been investigated to explain the effects of oxidative stress on bipolar disorders, and this might be reason why there are conflicting results in various studies which have been mentioned above. Further, the patients with bipolar disorder in remission during this study, they might have been in different episode in the past.

In this study, no statistically significant difference was detected between oxidative parameters of bipolar disorder patients in remission and healthy controls. To the best of our knowledge, this is the first study ever to report the levels of IMA in bipolar disorder and to compare those with healthy controls. Further investigations are needed to be carrying out to illustrate possible changes of oxidative balance in psychiatric disorders, including bipolar disorder. A more detailed evaluation of IMA on bipolar disorder is needed and various parameters are needed to be evaluated with more participants.

\section{Acknowledgment}

This study was presented as a poster presentation at the $41^{\text {st }}$ FEBS Congress September 2016 in Kusadasi, Turkey. We are grateful to the hospital staff who volunteered for this study.

\section{References}

1. Anderson IM, Haddad PM, Scott J. Bipolar disorder BMJ 2012; 345: e8508.

2. Yirün $\mathrm{MC}$, Yirün $\mathrm{O}$, Ünal $\mathrm{K}$, Yüksel RN, Altunsoy N. Serum TNF-related weak inducer of apoptosis (TWEAK) and TNF-related apoptosis-inducing ligand (TRAIL) levels of patients with bipolar disorder in manic episode, in remission and healthy controls. Psychiatr Res 2017; 257: 338-345.

3. Sies H. Oxidative stress: from basic research to clinical application. The Am J Med 1991; 91: 31-38.

4. Roy D, Quiles J, Gaze D, Collinson P, Kaski J. Role of reactive oxygen species on the formation of the novel diagnostic marker ischaemia modified albumin. Heart 2006; 92: 113-114.

5. Brown NC, Andreazza AC, Young LT. An updated metaanalysis of oxidative stress markers in bipolar disorder. Psychiatr Res 2014; 218: 61-68.

6. Williams JB. A structured interview guide for the Hamilton Depression Rating Scale. Archiv General Psychiatr 1988; 45: 742-747.

7. Akdemir A, Türkçapar M, Örsel S, Demirergi N, Dag I. Reliability and validity of the Turkish version of the Hamilton Depression Rating Scale. Comprehensive Psychiatr 2001; 42: 161-165.

8. Karadağ F, Oral ET, Aran Yalçın F, Erten E. Young Mani Derecelendirme Ölçeğinin Türkiye'de geçerlik ve güvenilirliği. Türk Psikiyatri Dergisi 2001; 13: 107-114.

9. Bar-Or D, Lau E, Winkler JV. A novel assay for cobaltalbumin binding and its potential as a marker for myocardial ischemia-a preliminary report. J Emerg Med 2000; 19: 311-315.

10. Takuma K, Baba A, Matsuda T. Astrocyte apoptosis: implications for neuroprotection. Progress Neurobiol 2004; 72: 111-127.

11. Reddy VS, Sethi S, Agrawal P, Gupta N, Garg R. Ischemia modified albumin (IMA) and albumin adjustedIMA (AAIMA) as biomarkers for diabetic retinopathy. Nepalese J Ophthalmol 2016; 7: 117-123.

12. Koç F, Erdem S, Altunkaş F, Özbek K, Gül EE. Ischemiamodified albumin and total antioxidant status in patients with slow coronary flow: a pilot observational study. Anadolu Kardiyol Derg 2011; 11: 582-587.

13. Banerjee U, Dasgupta A, Rout JK, Singh OP. Effects of lithium therapy on $\mathrm{Na}+\mathrm{K}+$-ATPase activity and lipid peroxidation in bipolar disorder. Progress NeuroPsychopharmacol Biol Psychiatr 2012; 37: 56-61.

14. Savas HA, Gergerlioglu HS, Armutcu F, Herken H, Yilmaz HR. Elevated serum nitric oxide and superoxide dismutase in euthymic bipolar patients: impact of past episodes. World J Biol Psychiatr 2006; 7: 51-55.

15. Versace A, Andreazza AC, Young L, Fournier JC, Almeida JR. Elevated serum measures of lipid peroxidation and abnormal prefrontal white matter in 
euthymic bipolar adults: toward peripheral biomarkers of bipolar disorder. Mol Psychiatr 2014; 19: 200-208.

16. Yirün MC, Kübranur Ü, Şen NA, Yirün O, Aydemir Ç. Evaluation of oxidative stress in bipolar disorder in terms of total oxidant status, total antioxidant status, and oxidative stress index. Archiv Neuropsychiatr 2016; 53: 194.

17. Kunz M, Gama CS, Andreazza AC, Salvador M, Ceresér KM. Elevated serum superoxide dismutase and thiobarbituric acid reactive substances in different phases of bipolar disorder and in schizophrenia. Progress NeuroPsychopharmacol Biol Psychiatr 2008; 32: 1677-1681.

18. Andreazza A, Cassini C, Rosa A, Leite M, de Almeida L. Serum S100B and antioxidant enzymes in bipolar patients. J Psychiatr Res 2007; 41: 523.
19. Kapczinski F, Dal-Pizzol F, Teixeira AL, Magalhaes PV, Kauer-Sant'Anna M. Peripheral biomarkers and illness activity in bipolar disorder. J Psychiatr Res 2011; 45: 156-161.

\section{*Correspondence to}

Kubranur Unal

Ankara Polatli Public Hospital

Ankara

Turkey 